\title{
ZQCD and universal matching to domain walls
}

\author{
C.P. Korthals Altes \\ Centre Physique Théorique, B.P.907 \\ Luminy, 13288 Marseille Cedex.
}

\begin{abstract}
We discuss the one loop matching procedure in ZQCD. Universality and Casimir scaling leavein terms of the 't Hooft coupling- just two combinations of parameters to be fixed numerically. These numbers are then the same for any number of colours.
\end{abstract}

\section{Introduction}

A 3d effective theory of QCD incorporating the center group symmetry has long defied the practitioners of thermal QCD.

The challenge is to marry in the model the non-perturbative nature of the centergroup [1] which involves large gauge transformations- and the perturbative nature of EQCD at high temperature. Recently Vuorinen and Yaffe [2] have furnished such a theory. This theory has the colour-magnetic gauge degrees of freedom (with field strength $F_{i j}$ ), and as matter field an $N \times N$ complex scalar field $Z=\frac{L}{N} \mathbf{1}+M$. The field $\mathrm{M}$ is traceless, so $L=\operatorname{Tr} Z$. The field $\mathrm{Z}$ is the Polyakov loop, but not anymore unitary.

Its unitarity is lost by averaging over space and integrating out the colour-electric modes of QCD. The field $M=H+i A$, with both $\mathrm{H}$ and A Hermitean and traceless, is a remnant of the latter modes. To garantuee the resurgence of the massless fourth component of the vector potential (in our case A) the theory is supposed to have a large global $S U(N) \times S U(N)$ invariance, broken to the diagonal $\mathrm{SU}(\mathrm{N})$, which becomes the gauged colour group. Thermal effects are given by a potential $V_{1}$ and screen the fourth component of the vector potential so should break explicitely the global invariance but should obviously respect colour and $\mathrm{Z}(\mathrm{N})$ invariance. Transformation laws are

- Z(N) symmetry: $L \rightarrow z L, M \rightarrow z M$

- $S U(N) \times S U(N)$ symmetry: $M \rightarrow U M V^{\dagger}$.

Such a model can be used on the lattice to extract information on the hot phase of QCD, down to $T_{c}$. To have a straightforward continuum limit one would like to have superrenormalizable operators. 
Lattice calculations for any number of colours have taught us important features of QCD, so we want to keep $\mathrm{N}$ general.

This being said we can now write the action of the model:

$$
\begin{aligned}
S_{Z Q C D} & \left.=\frac{1}{g_{3}^{2}}(\operatorname{Tr} \overrightarrow{(D} Z)^{\dagger} \cdot \vec{D} Z+V_{0}+V_{1}+\operatorname{Tr} F_{i j}^{2}\right) \\
V_{0} & =-M_{Z}^{2} \operatorname{Tr} Z^{\dagger} Z+\frac{\Lambda}{N}\left(\operatorname{Tr} Z^{\dagger} Z\right)^{2}+\tilde{\Lambda} \operatorname{Tr}\left(Z^{\dagger} Z\right)^{2} \\
& +\Lambda_{d} \operatorname{det} Z+\text { c.c. }
\end{aligned}
$$

with $\vec{D}=\vec{\partial}+i\left[\vec{A}\right.$, and $F_{i j}=\partial_{i} A_{j}-\partial_{j} A_{i}+i\left[A_{i}, A_{j}\right]$. The dimensionful gauge coupling is $g_{3}$, and in this notation all fields and couplings have engineering dimensions as in four dimensions.

If $M_{Z}^{2}$ is positive we can set $Z=Z_{c} \mathbf{1}+g_{3} Z_{q}$, determine $Z_{c}$ by minimizing, and see that the field $\mathrm{A}$ is the Goldstone boson. Ignoring $V_{1}$ one has indeed zero mass for the field $\mathrm{A}$ and non-zero mass for all other fields.

$V_{1}$ contains radiative effects like Debye screening, so is $\mathcal{O}\left(\frac{g_{3}^{2}}{M_{Z}}\right)$. It can be split in a natural way into two parts $V_{1}=V_{U}+V_{Z}$. $V_{U}$ is superrenormalizable for all values of $\mathrm{N}$. By inspection one sees $V_{U}$ is $\mathrm{U}(1)$ invariant, instead of only $\mathrm{Z}(\mathrm{N})$ invariant. The other part is $V_{Z}$ containing all other possible operators which are colour singlet and $\mathrm{Z}(\mathrm{N})$ invariant. Explicitly:

$$
\begin{aligned}
V_{U} & =m_{M}^{2} \operatorname{Tr} M^{\dagger} M+\lambda_{M}\left(\operatorname{Tr} M^{\dagger} M\right)^{2}+\tilde{\lambda}_{M} \operatorname{Tr}\left(M^{\dagger} M\right)^{2} \\
& +\lambda_{M}^{\prime}\left|\operatorname{Tr} M^{2}\right|^{2}+\tilde{\lambda}_{M}^{\prime} \operatorname{Tr} M^{2} M^{\dagger^{2}} \\
& +\lambda_{L M} \operatorname{Re} L^{2} \operatorname{Tr}\left(M^{\dagger}\right)^{2}+\lambda_{L M}^{\prime} \operatorname{Re} L^{*} \operatorname{Tr} M^{2} M^{\dagger}
\end{aligned}
$$

whilst $V_{Z}=\nu_{N} \operatorname{Re} \operatorname{Tr} M^{N}+\ldots$ contains no $\mathrm{U}(1)$ invariants, only $\mathrm{Z}(\mathrm{N})$ invariants, and grows like the number of partitions of $\mathrm{N}$, and is non-superrenormalizable for $N \geq 5$.

The problem with this approach is a practical one already in the case of $\mathrm{N}=3$ : it has many unknown parameters in the potentials. The purpose of this note is to get more insight in the matching. In particular we note in eq. (9) that the effective potential giving rise to the domainwall shows, apart from Casimir scaling, independence of $\mathrm{N}$.

\section{Matching with EQCD}

EQCD is obtained from QCD by integrating out all modes with mass $\sim T$ :

$$
\begin{aligned}
S_{E Q C D} & =\frac{1}{g_{3}^{2}}\left(\operatorname{Tr}\left(\vec{D} A_{0}\right)^{2}+m_{E}^{2} \operatorname{Tr} A_{0}^{2}+\lambda_{E}\left(\operatorname{Tr}\left(A_{0}^{2}\right)^{2}\right.\right. \\
& \left.+\tilde{\lambda}_{E} \operatorname{Tr} A_{0}^{4}+\operatorname{Tr} F_{i j}^{2} \ldots\right),
\end{aligned}
$$

The EQCD parameters are to $\mathrm{O}\left(g^{4}\right)$ :

$$
m_{E}^{2}=\frac{g^{2} N T^{2}}{3}, \lambda_{E}=\frac{g^{2} N}{4 \pi^{2} N}, \tilde{\lambda}_{E}=\frac{g^{2} N}{12 \pi^{2}} .
$$

To this order $m_{E}=m_{D}$, the Debye mass. Matching to EQCD is done in the bulk vacuum by integrating out the heavy fields. Because of chiral invariance this gives only derivative terms. The matching is then immediate from $V_{U}$ (identifying $\mathrm{A}$ with $A_{0}$ ): 


$$
\begin{array}{r}
g_{3}^{2}=g^{2} T \\
m_{M}^{2}-\lambda_{L M} \tilde{v}^{2}=m_{E}^{2} \\
\lambda_{M}+\lambda_{M}^{\prime}=\lambda_{E} \\
\tilde{\lambda}_{M}+\tilde{\lambda}_{M}^{\prime}=\tilde{\lambda}_{E}
\end{array}
$$

\section{Matching to the walls}

The wall between $\mathrm{Z}(\mathrm{N})$ bulk vacua is characterized by a profile that interpolates the value of the Polyakov loop in one side and of the other side of the wall. What matters for the wall is only the phase difference so we can set without loss of generality the phase equal 1 and $\exp \left(i k \frac{2 \pi}{N}\right)$ in the two vacua.

First ignore $V_{1}$. Due to $S U(N) \times S U(N)$ invariance the interpolating field $Z_{w}$ can be written as $\frac{v}{N} U$ with $\mathrm{U}$ an $S U(N)$ matrix, provided $-\Lambda_{d}, \Lambda, \tilde{\Lambda} \geq 0 . U=\mathbf{1} \operatorname{resp} \exp \left(i k \frac{2 \pi}{N}\right)$ in the two vacua. In perturbation theory $v$ does not depend on where we are in the wall and equals the bulk VEV all the way through the wall. The gulley leading from one bulk vacuum to the other is flat. This degeneracy is lifted through fluctuations we now discuss.

The wall profile depends on $\mathrm{v}$ and the eigenphases of $\mathrm{U}$. That means it is a gauge independent quantity. To compute the wall and its free energy density we compute the path integral with the constraint that the eigenphases and $\mathrm{v}$ are fixed 4 . This gives us the gauge independent effective action $G\left(\left\{Z_{w}\right\}\right)$, and finally we minimize it with the apropriate boundary conditions on both sides of the wall thus fixing the wall profile $Z_{w}$.

There is a considerable simplification: we can choose

$$
Z_{w}=\frac{v}{N} \exp \left(i q Y_{k} \frac{2 \pi}{N}\right) .
$$

Here $\mathrm{q}$ is a variable that varies inside the wall from 0 to $2 \pi$.

$$
Y_{k}=\frac{1}{N} \operatorname{diag}(\underbrace{k, k, \ldots, k}_{N-k \text { times }}, \underbrace{k-N, k-N, \ldots, k-N}_{k \text { times }}) .
$$

is the generalized hypercharge and generates $\mathrm{Z}(\mathrm{N})$ elements $\left(1\right.$ and $\left.\exp i k \frac{2 \pi}{N}\right)$ on both sides of the wall, characterizing the bulk vacuum. With this choice the wall profile $Z_{w}=$ $\frac{L_{w}}{N} \mathbf{1}+M_{w}$ with $M_{w} \sim Y_{k}$.

To compute the fluctuations around $Z_{w}$ one sets $Z=Z_{w}+g_{3} Z_{q}$ and $\vec{A}=g_{3} \vec{Q}$. The terms linear in $Z_{q}$ are changed by the constraints on the eigenphases in the path integral. This change applies also to the masses of the fluctuating fields, because there is always a quadratic constraint on the eigenphases, like e.g. on $\operatorname{Tr} Z^{\dagger} Z$. Note that the mass of the transverse gauge field is induced by the wall in the kinetic term of the $\mathrm{Z}$ field, and that it is of the same order as the masses of all the other fluctuation fields.

To get rid of bilinear couplings of $\vec{Q}$ and $M_{q}$ induced by the wall, put $R_{\xi}$ gauge [3]:

$$
\mathcal{G}_{g f}=\frac{1}{\xi}\left(\vec{\partial} \cdot \vec{Q}+i \frac{\xi}{2}\left(\left[M_{w}^{\dagger}, M_{q}\right]+\left[M_{w}, M_{q}^{\dagger}\right]\right)\right)^{2} .
$$

For $\mathrm{N}=2$ (where $Z=\frac{\Sigma}{2}+i \Pi, \Sigma$ real, $\Pi$ Hermitean and traceless) this gauge condition produces the mass of the $\Pi^{ \pm}$components (due the gauge constraints) and of course that 
of the ghost and longitudinal fluctuations. Their common mass is $4 \xi v^{2} \sin (q / 2)^{2}$. So the corresponding three fluctuation determinants cancel and hence the $\xi$ dependence, as it should.

Only the determinant of the transverse potential, and eventually of remaining components $\Sigma_{q}$ and $\Pi_{q}^{3}$ of the $\mathrm{Z}$ field do contribute. They do mix inside the wall. The physical states have only background independent masses, hence their bulk values 0 and $\Lambda v^{2}$ (neglecting $\mathcal{O}\left(g_{3}^{2} / M_{Z}\right)$ contributions from $\left.V_{1}\right)$. Apart from the determinant also $V_{1}\left(L_{w}, M_{w}\right)$ contributes to the effective potential. One finds for the effective potential in one loop QCD respectively one loop ZQCD $\left(z^{\prime}=m_{D} z\right)$, using the results from section 2 ;

$$
\begin{aligned}
& G(q) / k(N-k) T^{3}=\frac{4 \pi^{2} T}{3 m_{D}} \int d z^{\prime}\left(\left(\partial_{z^{\prime}} q\right)^{2}+q^{2}(1-q)^{2}\right) \\
& G(q) / k(N-k) T^{3}=\frac{4 \pi^{2} T}{3 m_{D}} \int d z^{\prime}\left(\left(\partial_{z^{\prime}} s\right)^{2}+s^{2}(1-s)^{2}+c_{k} s^{3}+b_{k} s^{4}+z_{k} s^{6}+\ldots\right)
\end{aligned}
$$

where $s \equiv \frac{v}{N T} \frac{\sin (\pi q)}{\pi}$ in the effective action for ZQCD. This is the main result.

- Note Casimir scaling and universality: no $\mathrm{k}$ and $\mathrm{N}$ dependence respectively on the r.h.s.. Hence in ZQCD matching means $b_{k}=b, c_{k}=z_{k}=0$.

- Fluctuation determinants generate cubic terms. $c_{k} \equiv 0$ for $\mathrm{N}=2$. For $N \geq 3$ it has yet to be computed.

- $V_{U} / g_{3}^{2} N$ generates only terms in $b_{k}$.

- $V_{Z} / g_{3}^{2} N$ generates terms both in $b_{k}$ and for $N \geq 4$ in $z_{k}$.

\section{Conclusions}

Using the full gauge independent effective action for any number of colours N Casimir scaling and universality leave two quantities to be matched: $\mathrm{b}$ and $\frac{v}{N T}$. So for all $\mathrm{N}$ $b=1.60379 . .$. , and $\frac{v}{N T}=1.000811$.. from the $\mathrm{N}=2$ calculation in ref. [3]. The coefficients $b_{k}$, and $z_{k}$ have been computed to one loop order for $\mathrm{N}=2,3,4$ [4. The $c_{k}$ are yet to be computed for $N \geq 3$. The $\mathrm{SU}(2)$ (SU(3)) action has 5 (9) independent couplings and 4 (5) constraints. Higher loop matching may fix more coefficients [5. Lattice computations have for $N \geq 5$ less numerical control (non-superrenormalizability). The number of terms in $V_{Z}$ grows like the number of partitions of $\mathrm{N}$.

\section{References}

[1] R.D. Pisarski, Phys. Rev. D 62 (2000), 111501(R); Phys. Rev. D 74, (2006), 121703, hep-ph/0608242. A. Dumitru, R. D. Pisarski, Phys. Lett. B 504. 282 (2001).

[2] A. Vuorinen, L. G. Yaffe, Phys. Rev.D 74 025011, hep-ph/0604100

[3] Ph. de Forcrand, A.Kurkela, A. Vuorinen, hep-lat/0801.1566. Gauge constraints are neglected in this paper. A. Kurkela, Phys. Rev. D 76, 094507, 2007; hep-lat/0704.1416. A. Kurkela, these proceedings.

[4] C.P. Korthals Altes, Nucl. Phys.B420, 637 (1994), and to appear.

[5] Y. Schröder, these proceedings. 